\title{
REVISTA DEE ESTUDIOS INTERNAOCONALES El delta del Río Perlas, la integración regional y el desarrollo económico chino
}

\author{
Jorge Rafael Di Masi
}

Este trabajo apunta a estudiar las particularidades del área del Delta del Río Perlas como centro motor del desarrollo económico chino, a partir de una fuerte vinculación con sus áreas geográficas adyacentes, como Macao, Hong Kong o Taiwán, así como con el resto de la región y del mundo. China es mucho más que el territorio continental, y constituye una vasta red de vinculaciones humanas, comerciales de inversión entre territorios con diferente status jurídico. Alli aparecen diversos actores que aportan en un mismo sentido a la construcción de proyectos individuales y colectivos más o menos homogéneos, que cambian en forma permanente la configuración del poder regional, en este caso a partir de una fuerte vinculación entre los distintos territorios que componen el Gran Delta del Río Perlas. En este proceso, se ha ido construyendo gradualmente una red de intercambios e interacciones a todo nivel -tanto privado como público- que de alguna manera obliga a los actores a adaptarse a la nueva realidad y promover acciones novedosas, tales como la firma de los CEPA (Closer Economic Partnership Arrangement), que han modificado así parcialmente el rumbo del esquema de integración regional. La posición del Delta es estratégica, puesto que es una de las ventanas de China al mundo y además genera un polo de atracción muy importante, lo que nos obliga a evaluar seriamente el curso que ha de seguir el extraordinario proceso de desarrollo económico que China protagoniza actualmente y que la han convertido en una de las grandes potencias mundiales.

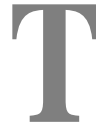

\section{INTRODUCCIÓN}

odos los estudios actuales relativos a China comienzan destacando los extraordinarios logros que este país ha tenido en las últimas décadas y el significado que tienen como determinantes del futuro del sistema internacional. Y no está mal hacerlo. Sin lugar a dudas, este país que por razones que se analizarán más 
adelante es mucho más que el territorio de la República Popular China (RPCh), llama hoy la atención del mundo y concita el interés de diversos círculos que se han lanzado a intentar conocerla con mayor profundidad.

\section{China es mucho más que un territorio delimitado por una frontera y con una bandera que lo simboliza.}

Como parte de esa posibilidad de reflexionar sobre China -que en esta ocasión nos brinda la Universidad de Chileintentamos incorporar una variable diferente a través de la cual podamos acercarnos, ya no a través del todo sino de una de sus partes. Como afirmamos en el primer párrafo, China es mucho más que un territorio delimitado por una frontera y con una bandera que lo simboliza. China es una antigua civilización que se ha expandido por una gran área geográfica, es la Revolución de 1949, también es los chinos de ultramar, así como los territorios cuya soberanía estuvo o está en disputa. Muchos ejemplos más se podrían dar para definir China y su número sería infinito. Este ejemplar de la Revista de algún modo reflejará también esa diversidad.

El objeto de este trabajo, en consecuencia, será estudiar las particularidades del área del Delta del Río Perlas como centro motor del desarrollo económico chino, a partir de una fuerte vinculación con sus áreas geográficas adyacentes, como Macao, Hong Kong o Taiwán, así como con el resto de la región y del mundo.

El Delta ha funcionado desde hace varios siglos como escenario natural de los intercambios comerciales entre mercaderes. También fue puerta de vinculación de China con el mundo. El movimiento de personas, productos y capitales en la zona se registra desde tiempos ancestrales, cuando los medios de transporte eran mucho más rudimentarios. Hoy es una de las zonas que cuenta con una infraestructura de transporte y comunicaciones que se modifica día a día para atender a la extraordinaria demanda que le imprimen los negocios internacionales.

A medida que el gobierno central de la República Popular China comenzó a recuperar territorios que antes estaban controlados por potencias europeas, como Hong Kong en 1997 y Macao en 1999, se aceleró la dinámica de esta subregión debido a que se facilitaron los intercambios, la legislación se modificó y el área empezó a disfrutar de las medidas transformadoras que China incorporó en su política económica durante las últimas tres décadas.

Además, el crecimiento de la actividad económica la convirtió en un centro de inmigración para los campesinos chinos que buscan mejorar su situación empleándose en las miles de fábricas abiertas en la zona.

De un tiempo a esta parte se ha desarrollado el concepto del Gran Delta del Río Perlas (GPRD), mediante el cual se pretende institucionalizar de alguna manera este relacionamiento desarrollando planes conjuntos de infraestructura y promoción de la inversión local. El GPRD incluye oficialmente parte de la provincia de Guangdong (los municipios de Dongguan, Foshan, Guangzhou, Huizhou, Jiangmen, 


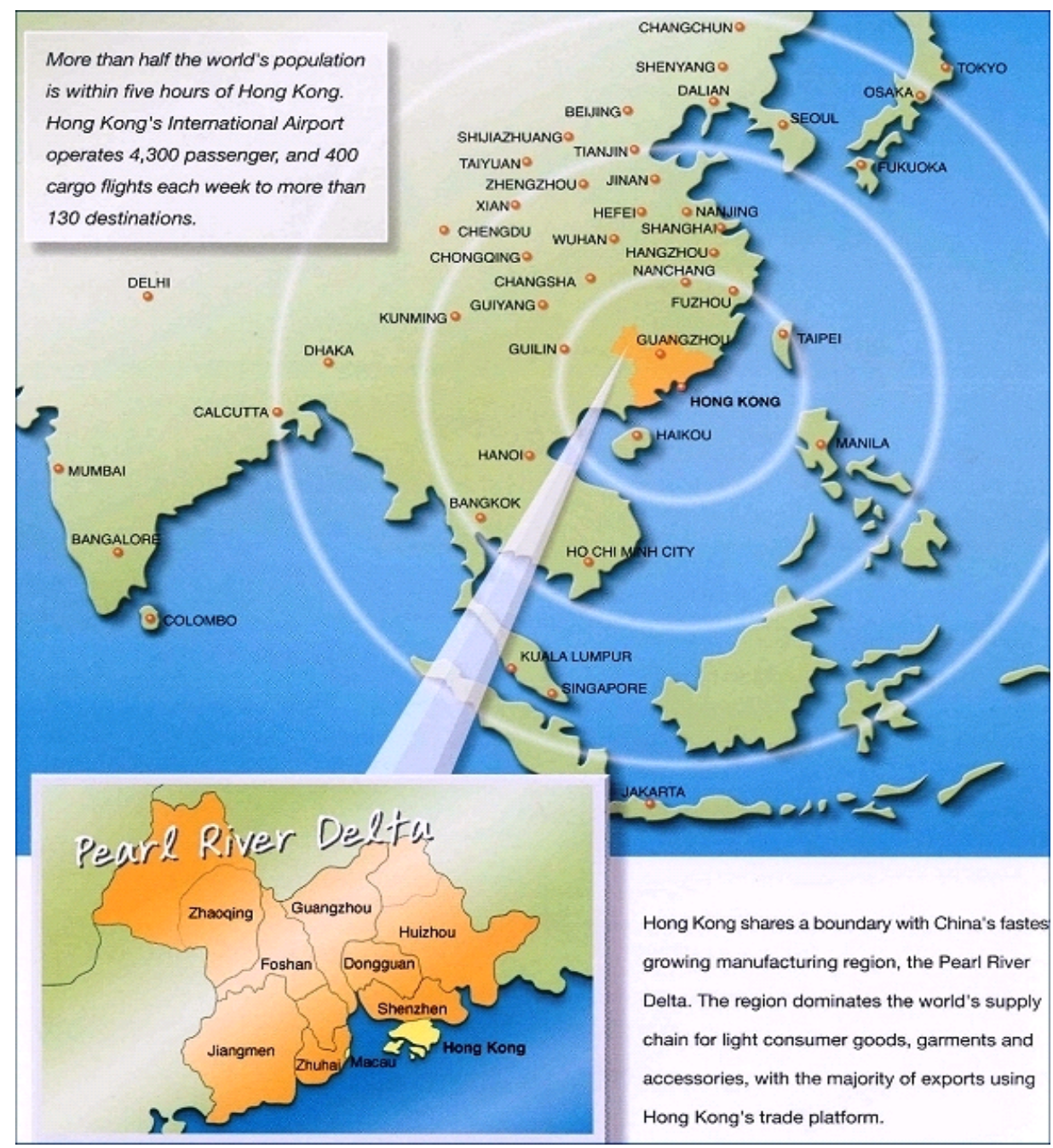

Fuente: www.gprd.com

Shenzhen, Zhaoquing, Zhongshan y Zhuhai) y las Regiones Administrativas Especiales de Macao y Hong Kong.

La superficie total del Gran Delta es de 41.294 kilómetros cuadrados, que en un 97\% corresponden a territorio continental y solo un 3\% a Hong Kong y Macao. Su población es de 48 millones de habitantes, de los cuales 41 millones viven en el conti- nente, 7 millones en Hong Kong y 440.000 en Macao. Su ubicación geográfica privilegiada lo convierte en un verdadero centro geográfico y económico de referencia para todo el este asiático, como lo muestra el mapa que figura en esta página.

Es interesante analizar como se fue construyendo esa vinculación intra subregional en la cual Hong Kong y Macao 
actuaban como proveedores de capital, y Guangdong de mano de obra de bajo costo, territorio y recursos naturales. Esos factores se conjugaron para producir una verdadera revolución dentro de China, convirtiéndose hoy esta zona en el área más importante de producción manufacturera del mundo.

Esta interacción, que capitaliza las ventajas que ofrece cada uno de los territorios nombrados, se caracteriza por una dinámica en la cual el capital privado tiene un rol fundamental. Sin embargo, como era de esperar, los primeros pasos de promoción correspondieron al sector público que mediante distintas medidas fue estimulando a los sectores privados a que aprovecharan los incentivos fiscales, la infraestructura y otros elementos positivos para consolidar una verdadera alianza entre lo privado y lo público en beneficio de la región.

Se puede afirmar que si bien hoy ese equilibrio se mantiene, el sector privado ha adquirido una dinámica propia que lo convierte en el gran motor de la profundización de las relaciones dentro del área y de su posterior internacionalización.

De los tres territorios, el que mayor peso tiene en la determinación de las políticas de fortalecimiento del GPRD es, sin dudas, el gobierno provincial de Guangdong. Por el hecho de ser provincia y no una Región Administrativa Especial como Macao y Hong Kong, el poder político de Guangdong es mucho mayor y determinante para la inserción del área en la política de la RPCh. Justamente aquí aparece una de las cuestiones centrales para el desarrollo futuro del área: cómo se desa- rrollará en el futuro la relación con el poder central de Beijing y otras regiones muy influyentes, como Shanghai.

\section{El poder político de Guangdong es determinante para la inserción del área en la política de la RPCh.}

\section{Algunas CONSIDERACIONES SOBRE LAS} RELACIONES ENTRE GUANGDONG Y BEIJING

Desde la creación de la RPCh, la provincia de Guangdong continuó siendo como era tradicional, la mayor puerta de relación de China con el mundo. Este carácter distinguió a Guangdong como una zona que siempre se sintió diferente del resto de China. La propia geografía, su posición tan al sur, la mantuvo alejada de las influencias provenientes de los grandes movimientos del norte o, en todo caso, cuando las adaptaron, mantuvieron sus propias características.

Aun dentro de Guangdong existe una diversidad social que se refleja en que las comunidades que habitan la provincia, suelen relacionarse de maneras diferentes. Uno de los ejemplos más claros es que si bien el cantonés es el idioma dominante, se hablan varios dialectos de este y otros idiomas como el hakka, el hokkien, y otros.

El propio carácter de los habitantes de Guangdong, influido por su alejamiento de las capitales del norte lo hace estar orgullosos del nivel de autarquía que poseyeron desde tiempos antiguos, y reivindican los más de quince gobiernos inde- 
pendientes que tuvieron desde su incorporación al Imperio Chino en el año 214 antes de Cristo. Es cierto también que esos gobiernos nunca propusieron la independencia del poder central, sino que actuaron con un nivel de autonomía mayor, lo que generó ese sentimiento en sus habitantes. El único intento de establecer una identidad política distinta fue el del terrateniente local Chen Jitang en la década de 1920 y 1930.

\section{Las cifras corresponden al cambio estructural de la economía de la zona caracterizado por la caída de la actividad agrícola, el aumento de las exportaciones y el desarrollo de la industria liviana.}

El Partido Comunista Chino de Guangdong tuvo una distante relación con los líderes que gobernaban desde Beijing, tanto que en 1952 el Comité Central envió una misión para eliminar todos los cuadros locales y reemplazarlos por «norteños». Años después, en 1957, los antiguos militantes del Partido local, organizaron un levantamiento armado contra los del norte que fue derrotado y significó la eliminación definitiva de todos los líderes que habían dirigido el Partido antes de 1949.

De este modo, el gobierno central chino retomó el control de la zona, no sin antes otorgar algunas ventajas -que sirvieran como bálsamo-en la distribución de los ingresos fiscales del gobierno provincial.

A partir de la reforma económica que comenzó en 1978, Guangdong parece ha- ber sido una de las zonas más beneficiadas con el cambio de escenario interno. Desde esa época, por ejemplo, el crecimiento económico de Guangdong superó en $3 \%$ el promedio nacional, y entre 1979 y 1991 creció un promedio 12, 6\%. En 1992, el ingreso per cápita era de 3440 yuanes, $68 \%$ más que el promedio nacional. Estas cifras responden a un cambio estructural de la economía de la zona que nace de una gran caída de la economía agrícola, un aumento de las exportaciones y un crecimiento explosivo de la industria liviana. Uno de los factores que potenció este cambio fue el masivo ingreso de inversión externa directa. Aquí desempeñó un papel fundamental el ingreso de capitales de los chinos de ultramar radicados en Taiwán, Hong Kong y Macao. Además hubo países como Japón que, en un proceso similar al que se dio en la década de 1980 y 1990, comenzó a relocalizar sus industrias en países de bajo costo laboral.

El inicio del boom económico de Guangdong se inició cuando el gobierno central permitió que el gobierno local buscara inversiones, y asistencia técnica y tecnológica fuera de la RPCh. En el año 1991, solo un 3\% del capital invertido fueron recursos del gobierno central. Esto tenía la doble ventaja de que Guangdong podía desarrollarse sin asistencia y liberaba al gobierno de Beijing importantes recursos que podían destinarse a otros fines.

Como elemento adicional, el gobierno provincial acordó un mecanismo de distribución de los ingresos muy favorable en virtud del cual debía entregar anualmente a Beijing una suma previamente fijada. En 
consecuencia, todo aumento de la actividad económica derivaría en un beneficio directo para Guangdong. Como el aumento se produjo, la provincia vio incrementados sustancialmente sus ingresos.

Al cabo de cinco años, el gobierno central indicó que debía revisarse el criterio aplicado y establecerse un porcentaje. Luego de algunas fricciones, se acordó modificar el sistema. Uno de los factores que llevó al gobierno central a promover el cambio fue también la postura de otros gobiernos provinciales que reclamaron contra la posición privilegiada de Guangdong.

\section{El gobierno central modificó el sistema de distribución de los ingresos que favorecía a Guangdong.}

Es interesante ver cómo, aunque son miembros del mismo Partido, en algunas circunstancias los funcionarios de Guangdong hacen valer la tradición autonomista que ha tenido la provincia a lo largo de los años. Claro está que ese autonomismo nunca ha llegado al extremo de plantear posturas secesionistas.

Uno de los momentos más trascendentes en el desarrollo de Guangdong se produjo cuando el gobierno central decidió -en el marco de la transformación estructural de su economía- crear las Zonas Económicas Especiales (ZEE). Las primeras fueron las de Shenzhen, frente a Hong Kong; Zhuhai frente a Macao y Shantou al otro lado del Estrecho de Taiwán. Su creación en el período 19791981 fue una de las medidas más acerta- das del gobierno central, ya que estos enclaves capitalistas pudieron acceder a enormes sumas de dinero por concepto de inversión externa directa, a la transferencia de tecnología a la empresas locales que en algunos casos se asociaban a las extranjeras y a la generación de puestos de trabajo mejor remunerados que en la actividad agrícola. Además, estas ZEE sirvieron de amortiguador tras la recuperación de Hong Kong y Macao. El temor de que ocurrieran migraciones masivas a estos dos territorios fue eliminado, pues aquellos podían encontrar allí un mejor destino con el que desenvolver sus actividades laborales en el territorio que ya conocían.

En ZEE se dio una mayor interacción entre los factores productivos locales ya descriptos antes: mano de obra y recursos naturales de la RPCh, capitales y tecnología de Macao y Hong Kong.

\section{El Delta Del Río PeRlas \\ Y LA INTEGRACIÓN REGIONAL}

En esta región se comprueba un caso más de lo que ha sido la característica propia de la integración en el este asiático. A pesar de que el control del proceso estaba básicamente en manos de un gobierno que llevó adelante una economía centralmente planificada, las características del país y de la región llevaron a que se diera una fuerte influencia de las fuerzas del mercado en la asignación de roles y la radicación de los factores productivos. Algo similar ocurrió en el resto de la 
región, donde la lógica del mercado y el modo de relacionamiento que se estableció entre los países asiáticos, sustentados en un proceso de rápido desarrollo económico, los hizo protagonizar una experiencia diferente, enmarcada dentro del paradigma de la «integración no convencional» ${ }^{1}$ o market integration ${ }^{2}$.

\section{La revaluación forzada del yen frente al dólar alteró significativamente la asignación internacional de los recursos.}

La construcción de este proceso no institucionalizado se basó en una serie de fuerzas económicas que confluyeron para estructurar una forma de relación de tipo vertical entre los países, no estática y que permitió a las naciones participantes ascender en la escala «social» regional.

El Profesor Naoki Tanaka definió la situación utilizando el concepto del flying geese pattern ${ }^{3} \mathrm{o}$ «modelo de desarrollo en cuña». Esta figura es una buena foto- grafía del proceso desarrollado en la región, en especial a partir de septiembre de 1985, luego del Acuerdo del Plaza, donde se decidió una revaluación forzada del yen frente al dólar, que alteró significativamente la asignación internacional de los recursos.

La apreciación del yen hizo que muchas empresas japonesas relocalizaran sus bases de producción en otros países donde los precios relativos de la mano de obra eran mejores: en una primero Corea y Taiwán, luego Hong Kong y Singapur -los cuatro miembros de la segunda generación de países que se incorporan al círculo virtuoso del desarrollo-y más adelante, cuando los dos primeros también fueron presionados por Estados Unidos para revaluar sus monedas, la onda expansiva de las inversiones se extendió a algunos países del ASEAN o de la tercera generación, como Malasia, Tailandia, Filipinas e Indonesia.

La clave para sostener este esquema de relaciones se basó en el dinamismo japonés para continuar su inversión en nue-

1 «...la región del Asia y el Pacífico constituye un bloque homogéneo bajo el liderazgo de Japón, que rompe con gran parte de los esquemas institucionalizados de la integración en otros sectores del planeta. Las perspectivas de ver materializada una Unión efectiva basada en estructuras jurídicas comunitarias, con la existencia de instituciones supranacionales que decidan políticas para todos sus miembros, parecen en esta Región virtualmente inalcanzables» . «La Cuenca del Pacífico: Un Modelo de Integración No Convencional», Jorge Rafael Di Masi y Pablo Martín Pinto, Serie Estudios, Nro.1, mayo 1993, Instituto de Relaciones Internacionales, Universidad Nacional de La Plata. La Plata, Argentina.

2 "The process is market driven, initiated by business enterprise, not by inter-governmental negotiation. There are no plans for monetary union, and little if any functional cooperation or formal harmonization by national governments». H.W. Arndt, «Anatomy of Regionalism» en Asia Pacific RegionalismReadings in International Economic Relations, compilado por Ross Garnaut y Peter Drysdale, Australia, Harper Educational Publishers, 1994.

3 Tanaka, Naoki, Policy Coordination in the Asia Pacific Region, Tokio, Institute of Developing Economies, 1990. 
vas tecnologías, que le permitió especializarse en la producción de bienes de capital y de consumo de alto valor agregado, mientras que los demás países producían grandes cantidades de bienes de consumo estandarizados, durables o no durables.

\section{La transferencia de tecnología y de los procesos de producción de Japón modificó la estructura industrial del este asiático.}

La estructura industrial del este asiático en su conjunto se desarrolló y su capacidad de suministrar bienes se incrementó gracias a la transferencia de tecnologías de producción y de los procesos de producción de Japón a los países de la segunda generación y luego a los de la tercera generación.

Como este fue un proceso dinámico e inclusivo, con el correr del tiempo algunos países de la segunda generación emprendieron caminos de mayor autonomización de su perfil productivo a partir de la inversión local en investigación para el desarrollo, infraestructura y educación. De este modo, hoy se observa que, por ejemplo, tanto Singapur como Hong Kong, que antes eran bases de actividades de montaje, se han especializado como centros de provisión de servicios al más alto nivel internacional. Su ubicación geográfica estratégica, como puerta de entrada al sudeste asiático el primero y a China el segundo, potenció la inversión estructural que hicieron sus gobiernos y les permitió prestar los mejores servicios aeroportuarios, portuarios, bancarios, financieros y de te- lecomunicaciones. En el caso de Corea y Taiwán, se han especializado más en el sector manufacturero, haciendo un especial hincapié en el desarrollo de nuevas tecnologías aplicadas a la producción. Corea, por ejemplo, compite palmo a palmo con Japón en la construcción de buques de carga y Taiwán se ha destacado por el desarrollo de semiconductores y productos informáticos en general.

Esta vinculación entre las tres generaciones de economías asiáticas creó un verdadero proceso de integración no institucionalizada, donde los flujos de comercio, inversiones y ayuda oficial para el desarrollo crecieron en forma sustancial, aumentando así el nivel de interdependencia regional

Esta integración no convencional funcionó a partir de las fuerzas del mercado, pero con respaldo del Estado, no en la construcción de estructuras legales y funcionales de integración, sino en el fomento de actividades de exportación y de inversión en el exterior, en otras palabras, de «asianización» de las empresas asiáticas, como otro aspecto de la internacionalización de las mismas. Esta asianización -o expansión intrarregional- es una de las caras de las políticas de promoción de exportaciones que aplicaron los países de la región para superar el modelo de sustitución de importaciones.

Si uno se preguntara por qué se expandieron por la región, las empresas japonesas primero, y las coreanas o taiwanesas después, la respuesta no sería que fue gracias a que sus gobiernos promovieron un proyecto de integración, con instituciones que los impulsaran a aprovechar las venta- 
jas concedidas por normas que establecieran áreas de preferencias arancelarias, zonas de libre comercio, uniones aduaneras o mercados comunes. La respuesta está en el rol desempeñado por la innovación tecnológica, la inversión en educación, la complementariedad respecto de la dotación de factores que los países y la empresas de la región supieron aprovechar.

\section{Las políticas de intervencionismo estatal crearon élites empresariales que sustentaron la expansión capitalista de los principales países de Asia.}

Puede resultar difícil comprender cómo se define un modelo de integración «no convencional» a partir de las fuerzas del mercado y, por otro lado, observar el grado de intervencionismo aplicado por los Estados desarrollistas de Asia. Sin embargo, en términos generales y porque en una región tan diversa, es difícil generalizar, puede afirmarse que las políticas de intervencionismo estatal de los principales países de Asia se cuentan entre las más eficientes registradas por la historia económica y que lograron crear élites empresariales que sustentaron su expansión capitalista. En muchos casos, el Estado intervino para impulsar «industrias nacientes» consideradas prioritarias mediante el desarrollo de planes estratégicos para programas de corto, mediano y largo plazo. Esto fue particularmente importan- te en las etapas de «puesta al día» que tuvieron sucesivamente los países de la región entre los años cincuenta y noventa. Además, la positiva intervención estatal creó las condiciones para la creación de grandes potencias exportadoras, como nos demuestra hoy la posición que ocupan los países de la región en el comercio y las inversiones globales.

Luego de esta experiencia, en la región se comprueba un intento por dar mayor uniformidad de criterios a la integración y avanzar en la creación de ciertas instituciones que permitan unificar criterios y acciones en pos de una profundización del proceso. Como afirma Chun Yang, de la Universidad de Hong Kong, "The market-driven regional economic integration of the Pearl River Delta (PRD) and Hong Kong, similar to most East Asian cases of economic integration, has occurred in the absence of a formal institutional framework. Since the late 1990s, however, institutions have been playing an active role in promoting economic integration in East Asia. In the context of the changing nature of regional integration in East Asia, this paper argues that institution-based economic integration has been emerging between mainland China (especially the PRD) and Hong Kong since the early 2000 s -twenty-five years after the ever-increasing socioeconomic interplay between the two began. This transition has occurred despite the unique framework of 'one country, two systems' adopted by transitional socialist China» 4 .

4 Chun, Yang, «From Market-Led to Institution-Based Economic Integration: The Case of the Pearl River Delta and Hong Kong», en Issues \& Studies, 40, N 2, junio de 2004, 9-118. University of Hong Kong. 
A partir de 2003, luego de la firma del CEPA (Closer Economic Partnership Arrangement) entre Hong Kong y el Gobierno Central de la RPCh se percibe un cambio en la forma de integrarse. El 1 de enero de 2004 entró en vigor el acuerdo que otorga acceso preferencial a los productos de Hong kong que quieran ingresar al continente y brinda facilidades a las empresas allí radicadas para invertir en Hong Kong. Más tarde se incorporó Macao a este mecanismo, completando lo que hoy se denomina el Gran Delta del Río Perlas.

\section{De acuerdo con el CEPA los productos de Hong Kong tienen acceso preferencial al continente.}

En los seis meses posteriores a la puesta en marcha del CEPA el intercambio aumentó a un ritmo notable. En ese período, las importaciones y exportaciones entre Guangdong, Hong Kong y Macao llegaron a 32.475 millones de dólares, es decir un crecimiento del $24.5 \%$, comparado con el mismo período del año anterior. Visto desde otro ángulo, ese volumen de comercio representó $20.4 \%$ del total del comercio exterior de Guangdong.

Las empresas de servicios de Hong Kong y Macao han participado activamente en la reforma y desarrollo de los sectores de logística, finanzas, convenciones y exhibiciones y servicios de consultoría en Guangdong. En el sector comercial, los productos fabricados en Hong Kong se venden masivamente en los centros comerciales de grandes urbes tales como
Guangzhou, Shenzhen, Zhuhai, Zhongshan, Foshan y Shantou. Según datos de 2005, en la provincia de Guangdong aperaban unos 560 negocios de personas físicas establecidas con capital de Macao y Hong Kong.

La implementación del CEPA, que significó facilitar los trámites para cruzar la frontera, llevó a un aumento de 27.25 en el número de personas ingresadas al continente por los distintos puestos fronterizos, mientras que el número de personas que se dirigieron del continente hacia Macao y Hong Kong creció un $94.1 \%$ en el período enero-mayo de 2004. Este exitoso primer semestre de 2004 también marcó un aumento de las inversiones de compañías de Hong Kong en Guangdong, que llegó a mil registros, un 52.7\% más que el año anterior.

En ese mismo año se acordó la firma del CEPA II que ampliaría la cantidad de productos elegibles para la aplicación del arancel cero. Inicialmente se había convenido que el 1 de enero de 2005 se pondría en marcha este esquema, pero luego se pospuso parcialmente hasta enero de 2006, fecha en que entró en vigencia plenamente. El número de productos aumentó de 374 en el CEPA a 1087 en el CEPA II.

Asimismo, en este marco se liberalizó la inversión en once sectores, tales como los servicios legales, contables, médicos, de distribución y de transporte. Si consideramos que la economía de Hong Kong está orientada al sector servicios, que ocupan un $90 \%$ de su producto interno bruto, el margen de crecimiento de esa alianza es muy elevado. 


\section{Conclusiones}

Este trabajo introductorio a la realidad de la subregión denominada Gran Delta del Río Perlas intentó mostrar una cara del extraordinario desarrollo de China y, en especial, de la región sudoccidental. Como se afirmaba al principio, mucho más que el territorio continental China es una vasta red de vinculaciones humanas, comerciales, y de inversión entre territorios con diferente status jurídico, en el que aparecen diversos actores que aportan en un mismo sentido a la construcción de proyectos individuales y colectivos. Estos proyectos, más o menos homogéneos, cambian permanentemente la configuración del poder regional, en este caso a partir de una fuerte vinculación entre los distintos territorios que componen el Gran Delta del Río Perlas.
Poco a poco se fue constituyendo una red de intercambios e interacciones a todo nivel -tanto privado como público- que de alguna manera obligó a los actores a adaptarse a la nueva realidad y a promover acciones novedosas como la firma de los CEPA, modificando así parcialmente el rumbo del esquema de integración.

Es claro que en el campo de la política la posición del Delta es estratégica, puesto que es una de las ventanas de China al mundo, y además genera un polo de atracción muy importante, visto con recelo y hasta envidia por otras áreas menos favorecidas del propio territorio de la RPCh. El área del Delta será, sin duda, uno de los puntos al que habría que poner atención para vislumbrar la trayectoria del extraordinario proceso de desarrollo económico que protagoniza actualmente China y que la ha convertido en una de las grandes potencias mundiales. 